(Aus dem Laboratorium der deutschen psychiatrischen Klinik [Prof. A. Pick] und dem pathologischen Institut [Prof. A. Ghon] in Prag.)

\title{
Kombination von multipler Sklerose und Syringomyelie.
}

\author{
Von \\ Otto Sittig, klin. Assistent. \\ Mit 3 Textfiguren.
}

(Eingegangen am 24. Juni 1914.)

Kombinationen verschiedener Erkrankungen des Nervensystems können selbstverständlich ebenso vorkommen wie solche anderer Erkrankungen und es läge demnach auch in der im Titel genannten Kombination nichts Erwähnenswertes vor, wenn nicht gerade sie zum Ausgangspunkt theoretischer Erörterungen und weitgehender Schlüsse über die Ätiologie der multiplen Sklerose gewählt worden wäre.

Außerdem ist diese Kombination eine immerhin recht seltene, so daß schon dieser Umstand allein die Mitteilung dieses Falles rechtfertigt.

Da die Ätiologie und Pathogenese der multiplen Sklerose noch recht dunkel ist und sichere Beweise für eine ätiologische Erklärung sich schwer erbringen lassen, so herrscht noch immer Streit, ob endogene oder exogene Faktoren für das Zustandekommen der multiplen Sklerose maßgebend sind.

Zur Lösung dieser Frage wurde nun auch das Vorkommen von Kombination von multipler Sklerose mit Bildungsanomalien des Zentralnervensystems, speziell mit Syringomyelie herangezogen.

In der Literatur sind mehrere derartige Fälle niedergelegt. Der Fall von Kiewlicz (Arch. f. Psych. XX, 1889) zeigte eine Kombination von multipler Sklerose mit Syringomyelie und Myelitis transversa. Der Autor will alle drei Erkrankungen auf ein Trauma zurückführen, das 3 Monate den ersten manifesten Symptomen der Erkrankung vorausging. Ferner erwähnt Strü m pell (Neurol. Centralbl. 1896) zwei Fälle der besprochenen Kombination und er will diesen Befund zugunsten jener Theorie verwerten, die eine endogene Ursache für die multiple Sklerose annimmt. Auch E. Müller (Die multiple Sklerose des Gehirns und Rückenmarks 1904) beschreibt einen hierher gehörigen Fall mit entsprechendem Sektionsbefund. In der Epikrise sagt Müller, der 
vor allem die endogene Theorie vertritt: „Ich sehe also in dieser Kombination von echter Sklerose en plaques mit einer sicherlich auf Entwicklungsstörungen beruhenden Form der Syringomyelie koordinierte Prozesse auf der gemeinsamen Basis einer angeborenen kongenitalen Veranlagung des Zentralnervensystems."

Ein einschlägiger Fall findet sich in Schlesingers Monographie über die Syringomyelie erwähnt (v. Ste ys kal). Sehr interessant ist der Fall Schüllers (Jahrb. f. Psych. XXVI, 1905), in dem multiple Sklerose mit Syringomyelie und Mikrogyrie sich kombiniert fand. Schüller meint, daß dieser Fall für die Ansicht Strü mpells spreche, „daß es Formen von multipler Sklerose gibt, die angeborenen Bildungsfehlern des Zentralnervensystems ihre Entstehung verdanken".

In der letzten Zeit hat aber die Theorie der exogenen Entstehung der multiplen Sklerose immer mehr Anhaltspunkte gewonnen, namentlich durch die pathologisch-anatomischen Untersuchungen, so daß Wohlwill in seinem großen Sammelreferate (Zeitschr. f. d. ges. Neur. u. Psych. Ref. VII) sagen kann, , ,daß über die exogene Natur und den vaskulären Ursprung der Erkrankung jetzt die Ansichten sich doch zu einigen und zu klären beginnen“".

Bei Besprechung der uns hier interessierenden Frage sagt Wohlwill: „Auf die Kombination mit Syringomyelie legte bekanntlich Str ü m pell großen Wert, da sie eine abnorme Gliaveranlagung bekunde. Aber einerseits vergleicht man hier doch, wenn man aufrichtig sein will, zwei Unbekannte miteinander, andererseits ist diese Kombination zu exzeptionell, um auf sie weitgehende Folgerungen zu bauen.“

Was die Ätiologie der Syringomyelie anlangt, so wird sie wohl in erster Linie als Entwicklungsanomalie aufgefaßt, doch sind auch andere Momente, wie Trauma, ja selbst Infektionskrankheiten für die Ätiologie herangezogen worden.

Im folgenden soll nun ein Fall von Syringomyelie und multipler Sklerose beschrieben werden, der im allgemeinen israelitischen Spital in Prag (Primarius Dr. Saar, dem ich für die freundliche Uberlassung der Krankengeschichte meinen Dank ausspreche) beobachtet wurde.

P. Stefanie, 21 Jahre, ledig, Kellnerin, wurde am 25. VI. $1913 \mathrm{im}$ Spital auf genommen. Sie soll vor 2 Jahren Typhus durchgemacht haben. Das jetzige Leiden soll erst seit einem halben Jahre bestehen und begann mit Schmerzen und Steifheit in den Beinen.

Status praesens: Pat. ist abgemagert, geht schwer, mit steifen Beinen, starker Intentionstremor der Hände, skandierende Sprache, Nystagmus, Patellarreflexe gesteigert, Fußphänomen vorhanden, Spasmen der unteren Extremitäten.

Es traten Schwindelanfälle auf. Nach 2 Monaten entwickelte sich eine Muskel. atrophie, besonders an den oberen Extremitäten, die Interossei verschwanden ganz, zeigten Entartungsreaktion. Tastsinn blieb normal, dagegen fehlte der Temperatursinn an den oberen und unteren Extremitäten. Es trat Inkontinenz der Blase 
und des Sphincter ani auf. Es bildete sich mehrfacher Decubitus, der schwer heilte. Die Reflexe waren immer gesteigert.

Die Sektion (Dr. Kraus) ergab ausgedehnte Decubitusgeschwüre am Kreuz und über beiden großen Trochanteren, hochgradige allgemeine Atrophie, eitrige Bronchitis der rechten Lunge mit eitriger Labulärpneumonie im Ober- und Unterlappen, geringer Milztumor, Degeneration der Organe.

Schädelumfang $49 \mathrm{~cm}$, Pachymeninx mäßig gespannt, durchscheinend, Leptomeninx zart getrübt, stark durchfeuchtet.

Das Gehirn normal konfiguriert, Gehirnwindungen, namentlich im Stirnhirn leicht verschmälert. Das Gehirn wurde unseziert in Formol fixiert. Auf verschiedenen Schnitten durch das 3 Tage in Formol fixierte Gehirn begegnet man mehreren grauroten und graugelblichen mehr oder weniger unscharf begrenzten Herden von maximal Hanfkorngröße, meist an der Rinden-Markgrenze.

Rückenmark: Der Zentralkanal im Cervikalmark, weniger im Thorakalmark umgewandelt in eine longitudinal verlaufende Höhle. Stellenweise eine grauweiß. liche Verfärbuug des Rückenmarks am Querschnitt in allen Höhen sichtbar.

Die mikroskopische Untersuchung ergab viele recht interessante Details, die auch für die Beantwortung der eingangs erörterten Frage von einiger Wichtigkeit sind.

Im Rückenmàrk fand sich eine quere spaltförmige Höhle, die durch das ganze Halsmark zog. An einer Stelle war diese Höhle durch einen schmalen Streifen Gewebes in zwei Höhlen geteilt. Die Höhle setzte sich nach unten bis zum untersten Dorsalmark fort, war in diesem Teile kleiner, mehr rundlich.

Halsmark: Die Querschnittsfigur bildet ein mit der längeren Achse quer gestelltes Oval. In der Mitte läuft der Quere nach eine schmale,

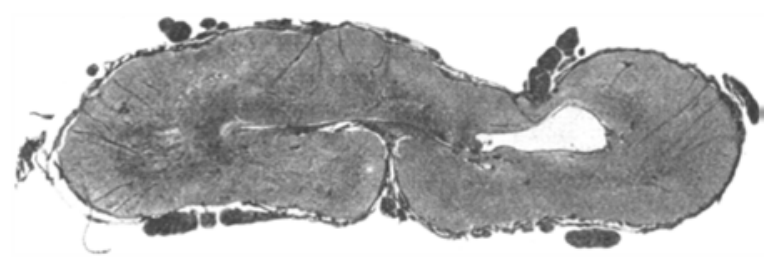

Fig. 1. Oberes Cervicalmark (van Gieson).

stellenweise spaltförmige Höhle (Fig. 1). In einer Partie sind zwei Höhlen vorhanden; an einzelnen Stellen ist ein spaltförmiger Zusammenhang dieser beiden Höhlen zu konstatieren. Die Höhle zeigt stellenweise einen Ependymbelag. An anderen Stellen ist sie von einer Bindegewebslage ausgekleidet. Dort wo zwei Höhlen bestehen, hat die eine kein Epithel, ist ganz von Bindegewebe ausgekleidet und schickt kurze divertikelartige Fortsätze aus.

Um die Höhle herum ist stark gewucherte Faserglia. Auch findet man größere bläschenförmige chromatinarme Gliakerne, stellenweise Anhäufung mehrerer Gliakerne. 
Das Markscheidenpräparat zeigt starken Ausfall in der grauen, sowie in der weißen Substanz. Die eine Hälfte des Rückenmarksquer. schnittes erweist sich als fast vollkommen marklos. Im Seitenstrang der andern Seite findet sich ein umschriebener Ausfallsherd.

Marchipräparate weisen Degenerationsherde in der weißen Substanz auf. In diesen Herden finden sich zahlreiche Körnchenzellen.

Die Gefäße sind etwas vermehrt und zeigen eine Bindegewebswucherung.

Oberes Dorsalmark: Querschnittsfigur noch etwas längsoval, in der Mitte eine längliche schmale Höhle, teilweise mit Ependymepithel

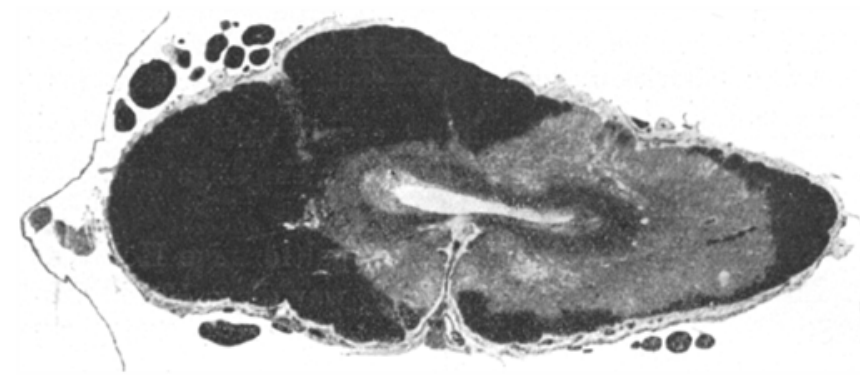

Fig. 2. Dorsalmark (Weigert).

(Fig. 2). Im übrigen wie Halsmark. Nur ist die Gefäßwucherung namentlich um den erweiterten Zentralkanal hier stärker. Die Adventitialzellen zeigen progressive Veränderungen, längliche bläschenförmige Kerne.

Um die Gefäße findet sich eine Vermehrung der Gliakerne. Vereinzelt finden sich Gliazellen, deren Plasma sich deutlich färbt, die einen oder zwei, selten mehrere bläschenförmige, helle Kerne haben.

Unteres Dorsalmark und Lendenmark: Keine Höhlenbildung mehr.

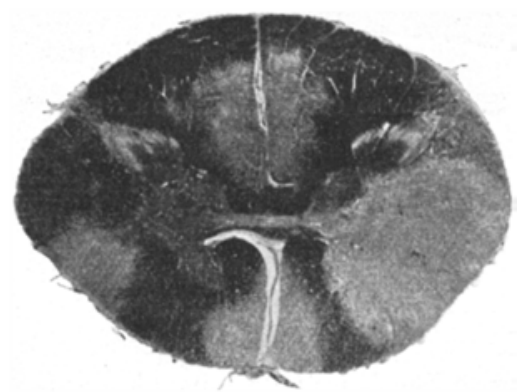

Fig. 3. Unterstes Dorsalmark (Weigert).

Gliawucherung und herdförmiger Markscheidenausfall in der weißen Substanz (Fig. 3).

Sekundäre Degeneration fand sich nirgends im Rückenmark. 
Im Gehirn fanden sich mehrfach Herde, die wir vielleicht in folgender Weise gruppieren können.

1. Längliche streifenförmige Markherde mit starker Wucherung der Faserglia und der Gliazellen. Einzelne enthalten zahlreiche Körnchenzellen und Fettsäurekrystalle.

2. Große unregelmäßig begrenzte Herde, die vom Mark auch auf die Rinde übergreifen und offenbar durch Konfluieren kleinerer Herde entstanden sind.

3. Kleine rundliche Herde von Markfaserausfall an der Rindenmarkgrenze.

4. Keilförmige Rindenherde. An einer Stelle des Occipitallappens finden sich an zwei benachbarten Windungen zwei symmetrische keilförmige Ausfallsherde.

5. Kleine rundliche Rindenherde ganz analog dem fleckweisen Markfaserschwund (Fischer) bei der Paralyse.

Interessant war in diesem Falle das Verhalten der Gefäße. In vielen Herden und deren Umgebung waren um Gefäße kleinzellige Infiltrate, aus Lymphocyten und Plasmazellen bestehend

Fassen wir den Fall zusammen, so ist zu sagen, daß $1^{1} / 2$ Jahre nach einem Typhus die ersten manifesten Erscheinungen der multiplen Sklerose auftraten, die dann zu dem typisehen Symptomenkomplexe von multipler Sklerose führten, der aber durch Atrophie an den oberen Extremitäten und Thermanästhesie kompliziert war. Die Untersuchung des Zentralnervensystems ergab den Befund einer multiplen Sklerose und Syringomyelie. Die Syringomyelie war bemerkenswert durch die stellenweise ziemlich erhebliche Gefäßneubildung und Bindegewebswucherung, wie sie von Thomas und Hauser beschrieben wurde. Die multiple Sklerose war im Gehirn and Rückenmark verbreitet.

Daß im Rückenmark tatsächlich eine Kombination beider Erkrankungen vorliegt, geht daraus hervor, daß im unteren Dorsal- und Lendenmark ganz umschriebene Herde von Markfaserausfall in der weißen Substanz vorkommen (vgl. Fig. 3). Aber auch im Halsmark, wo der syringomyelische Prozeß im Vordergrund steht, sehen wir in dem einen Seitenstrang einen ganz umschriebenen Ausfallsherd. Es unterliegt also keinem Zweifel, daß diese Herde solche einer multiplen Sklerose sind. Man hätte nämlich daran denken können, daß es sich dort, wo die Gliose vom Zentrum ausgehend bis an den Rand des Rückenmarks reicht, um ein Ubergreifen der zentralen Gliose auf die weiße Substanz handeln könnte; die umschriebene Form mancher Herde beweist aber, daß diese Herde mit der Syringomyelie nichts zu tun haben, sondern der multiplen Sklerose zukommen.

Die Gehirnherde zeichneten sich durch entzündliche Gefäß- 
infiltrate, durch starke Wucherung der Gliazellen und -fasern, manche durch Körnchenzellenbildung aus.

Nach diesen Merkmalen müssen wir diese Herde wohl zu den a k u teren der multiplen Sklerose rechnen.

Was die Verwertung unseres Falles für die eingangs erörterte Frage anlangt, so kann zwar aus ihm weder für die eine noch für die andere Theorie ein strikter Beweis gezogen werden; doch scheint uns dieser Fall besonders schön zu zeigen, wie schwierig die Deutung derartiger Fälle und ihre Verwertung für die Lösung theoretischer Fragen ist.

In unserem Falle war vor $1 \frac{1}{2}$ Jahren eine Infektionskrankheit - Typhus - vorausgegangen. Im Gehirn ist dabei der Prozeß ein recht frischer. Im Rückenmark allerdings scheinen die Veränderungen, die die multiple Sklerose gesetzt hat, schon älter zu sein.

Die Syringomyelie ist auch insofern bemerkenswert, als Gefäßveränderungen und Vermehrung, sowie Bindegewebswucherung, vorhanden sind (néoplasie conjonctivo-vasculaire Thomas und Ha user).

Wenn wir also auch keinen zwingenden Beweis erbringen können, daß die multiple Sklerose in ursächlichem Zusammenhang mit dem Typhus steht, so müssen wir doch andererseits sagen, daß diese mehr akute Form der multiplen Sklerose gar keine Kriterien der Entwicklungsanomalie an sich trägt. Und mit Bezug auf die Syringomyelie werden wir an die Worte Wohlwills erinnern, die unsere Unkenntnis der Ätiologie der Syringomyelie betonen.

Wenn man auch in dieser Hinsicht sehr zurückhaltend sein muß und keine weittragenden Schlüsse wird ziehen können, so wird man doch, namentlich aus dem histologischen Verhalten der Hirnherde, die eine entzündliche Gefäßinfiltration aufwiesen, sagen müssen, daß unser Fall von Kombination einer multiplen Sklerose mit Syringomyelie keinen Beweis für die endogene Entstehung der multiplen Sklerose liefern kann.

Wenn man auch die Auffassung, die Syringomyelie sei eine Entwicklungsstörung, als richtig anerkennt, so kann man aus deren Kombination mit multipler Sklerose in unserem Falle noch nicht auf eine gleiche Genese der letzteren schließen. 